\title{
EGFR-TKIs in adjuvant treatment of lung cancer: to give or not to give?
}

This article was published in the following Dove Press journal:

OncoTargets and Therapy

13 October 2015

Number of times this article has been viewed

\author{
Aleksandar Milovancev' \\ Vladimir Stojsic ${ }^{\prime}$ \\ Bojan Zaric' \\ Tomi Kovacevic' \\ Tatjana Sarcev' \\ Branislav Perin' \\ Konstantinos Zarogoulidis ${ }^{2}$ \\ Katerina Tsirgogianni² \\ Lutz Freitag ${ }^{3}$ \\ Kaid Darwiche ${ }^{3}$ \\ Drosos Tsavlis ${ }^{2}$ \\ Athanasios Zissimopoulos ${ }^{4}$ \\ Grigoris Stratakos ${ }^{5}$ \\ Paul Zarogoulidis ${ }^{2}$ \\ 'Institute for Pulmonary Diseases \\ of Vojvodina, Clinic for Thoracic \\ Oncology, Faculty of Medicine, \\ University of Novi Sad, Serbia; \\ 2Pulmonary Department-Oncology \\ Unit, "G Papanikolaou" General \\ Hospital, Aristotle University of \\ Thessaloniki, Thessaloniki, Greece; \\ ${ }^{3}$ Department of Interventional \\ Pneumology, Ruhrlandklinik, \\ University Hospital Essen, University \\ of Duisburg-Essen, Essen, Germany; \\ ${ }^{4}$ Nuclear Medicine Department, \\ Democritus University of Thrace, \\ Alexandroupolis, ${ }^{5}$ Department of \\ Interventional Pneumology, "Sotiria" \\ Hospital of Chest Diseases, Athens, \\ Greece
}

Correspondence: Paul Zarogoulidis Pulmonary Department-Oncology Unit, "G Papanikolaou" General Hospital, Aristotle University of Thessaloniki,

Exohi 57010, Thessaloniki, Greece

Tel +30697727 I974

Fax +30 2310992424

Email pzarog@hotmail.com
Abstract: Epidermal growth factor receptor-tyrosine-kinase inhibitors (EGFR-TKIs) brought a significant revolution in the treatment of non-small-cell lung cancer (NSCLC). In a short period of time, EGFR-TKIs became the standard of treatment for mutation-positive, advanced stage non-squamous NSCLC. In recent years, second- and third-generation EGFR-TKIs are emerging, further widening the clinical use. However, the question of EGFR-TKIs efficiency in the treatment of early stage NSCLC still remains open. Early clinical trials failed to approve the use of EGFR-TKIs in adjuvant setting. The majority of these early trials were performed in unselected NSCLC populations and without standardized biomarker identification. One should certainly not rely solely on these results and dismiss the use of EGFR-TKIs as adjuvant therapy. Many important questions are still unanswered. Most important issues such as stage heterogeneity (IA-IIIA), timing (after or concomitantly with chemotherapy), and type of administration (monotherapy or combination) need to be answered in near future. Adjuvant TKIs in the treatment of lung cancer might offer significant number of advancements. Having in mind the significant duration of response observed in advance disease setting, there could be place for prolongation of response in adjuvant setting potentially, leading to improvement in survival. TKIs could offer less-toxic adjuvant treatment with better efficiency than chemotherapy. However, there is a chronic lack of randomized controlled trials in this field, leading to inability to draw any scientifically sound conclusion with regard to the adjuvant treatment. For now, the use of EGFR-TKIs outside clinical trial setting is not recommended. The purpose of this review is to evaluate current and available data.

Keywords: adjuvant chemotherapy, EGFR-TKIs, erlotinib, gefitinib, NSCLC

\section{Introduction}

Lung cancer is the most common cancer and the most common cause of cancer death in males, while it is a fourth most commonly diagnosed cancer and the second leading cause of cancer death in females. ${ }^{1}$ The biggest challenge of modern oncology is definitely improving overall survival (OS), bearing in mind that 5-year survival rate remains $<15 \%$ among all stages of the disease. ${ }^{2}$ Surgery continues to be the best curative treatment option for the patients with non-small-cell lung cancer (NSCLC). Unfortunately, only a small fraction of patients are eligible for surgical resection with disappointing 5 -year survival rates of $67 \%$ and $23 \%$ for stage IA and stage IIIA, respectively. ${ }^{3}$ In general, the main problem for achieving long-term survival is tumor recurrence. ${ }^{4}$ In other words, so far we were unable to accomplish sustainable duration of response. It is known that cisplatin-based chemotherapy given in a neo-adjuvant or adjuvant setting can modestly contribute to the prolongation of survival. The Lung Adjuvant Cisplatin Evaluation meta-analysis showed statistically significant benefit of OS and disease-free survival (DFS) for patients who received adjuvant chemotherapy. ${ }^{5}$ The introduction of epidermal growth factor receptor-tyrosine-kinase inhibitors 
(EGFR-TKIs) was the breaking point in the treatment of advanced EGFR-mutated NSCLC. Indeed, multiple randomized Phase III trials have showed that patients treated with EGFR-TKIs had an outstanding response rate (58\%-75\%) and more than doubled progression-free survival in comparison to patients on standard chemotherapy. ${ }^{6,7}$ Superiority of EGFR-TKIs over the standard chemotherapy in patients with advanced EGFR-positive NSCLC reasonably imposed question: whether these agents can be useful for patients with earlier stages of NSCLC? Currently, there is evidence that targeted therapy has a significant impact on OS and DFS in other tumor types. Adjuvant trastuzumab notably improves OS in resected HER2-positive breast cancer, ${ }^{8}$ while adjuvant imatinib-mesylate significantly improves DFS in patients with resected gastrointestinal stromal tumor (GIST), positive for KIT protein, compared with placebo group $(\mathrm{HR}=0.35$; $P<0.0001) .{ }^{9}$ The aim of this article is to elaborate present status of adjuvant treatment with EGFR-TKIs in NSCLC.

\section{Adjuvant TKIs treatment for early stage NSCLC \\ Early trials of adjuvant TKIs in unselected NSCLC populations}

The beginning of the investigation of adjuvant EGFR inhibition in resected NSCLC is dated about a decade ago with two large randomized trials designed to assess EGFR-TKIs in early stage NSCLC. Both trials were conducted in an unselected patient population. The first one was SWOG S0023, a Phase III study that evaluated inoperable patients with stage III NSCLC who were expected to receive concurrent chemoradiation. Patients without disease progression after that treatment were randomized to receive either gefitinib or placebo for 2 years. ${ }^{10}$ The primary objective of this study was OS. The study enrolled 243 of the planned 840 patients (118 patients were assigned to the gefitinib arm, and 125 patients were assigned to placebo), and unfortunately, it was interrupted by an unplanned early analysis because of the results from the ISEL trial. ${ }^{11}$ The ISEL trial was a randomized study done in metastatic patients evaluating the effect on survival of gefitinib as a second- or third-line treatment for patients who were refractory to or intolerant of their most recent chemotherapy regimen. Surprisingly, this trial had negative results and verified no survival benefit with gefitinib. At that time, all other trials using gefitinib were inspected for safety analysis. SWOG 0023 study, unfortunately, showed that patients on gefitinib treatment were doing worse compared with those on placebo (with a median follow-up time of 27 months and median survival time of 23 months for gefitinib and 35 months for placebo; $P=0.013$ ); the results were enough for premature termination of the trial. The second large randomized Phase III trial was BR.19 trial, investigating EGFR-TKIs in the adjuvant setting for completely resected NSCLC. ${ }^{12}$ In this study, patients with complete resection of stage IB-IIIA NSCLC and optional adjuvant chemotherapy were randomized to receive either gefitinib or placebo for 2 years. This study was stopped soon after the ISEL study was found to be negative, as well as the aforementioned SWOG 0023 interim report. Of a planned 1,160 patients, only 503 were enrolled (251 to gefitinib and 252 to placebo) with the median duration of the study treatment $<5$ months. OS, as a primary endpoint of this study, showed no statistically significant difference between placebo and gefitinib $(\mathrm{HR}=1.23 ; P=0.136)$. Among patients with known EGFR status, 344 patients had wild-type status, where only 15 (4\%) had mutations (all adenocarcinoma). The subset analysis for those 15 patients with EGFR-positive tumors (seven on gefitinib and eight on placebo) showed no beneficial effect of gefitinib on DFS $(\mathrm{HR}=1.84 ; P=0.40)$ or OS $(\mathrm{HR}=3.16 ; P=0.15)$. Therefore, the BR.19 study is underpowered, because it was prematurely stopped. Also, $<50 \%$ of the planned number of patients was enrolled. The patients were planned to be treated for 2 years, and actually, they received treatment for $<5$ months on average, which is considered suboptimal duration of therapy. A Group of Chinese authors analyzed the BR.19 study retrospectively and pointed out several possible explanations for such poor results. ${ }^{13}$ First, the median time of treatment was shorter than usual leaving not enough space for TKIs to demonstrate their efficacy. Second, a low EGFR mutation rate was observed in this study $(15 / 503,3.0 \%)$ most likely due to nonselected population. However, there is also a hypothesis that there might be a lower EGFR mutation rate in early stages of NSCLC and that, in general, early and advanced NSCLC might have different biologic characteristics. Considering those facts, it is impossible to give conclusions regarding the efficacy of adjuvant TKIs for patients with EGFR-positive NSCLC.

\section{Adjuvant TKI treatment in selected EGFR-positive populations}

In 2011, a group of authors from the Memorial SloanKettering Cancer Center (MSKCC) offered promising data, showing for the first time that patients with NSCLC after surgery can benefit from adjuvant TKIs. ${ }^{14}$ They retrospectively collected 167 patients with EGFR-positive completely resected lung adenocarcinomas and divided them into two 
groups: group of patients who were not treated with TKIs (111 patients) and group of patients who were on TKIs (56 patients). Then authors analyzed both groups of patients as separate cohorts and compared them in terms of outcome. Many of these patients received adjuvant standard chemotherapy in addition to TKI treatment. This group reported impressive 89\% 2-year DFS rate as compared with 2-year DFS rate $(72 \%)$ for patients who did not receive adjuvant TKI (trend toward significance $P=0.06, \mathrm{HR}=0.53$ ). These data suggest that there might be a benefit in delaying recurrence of disease. Additionally, authors reported OS, which did not look statistically significant; although at the time of the initial report in 2011, median OS rate had not yet been reached. The same group of authors from MSKCC, in late 2012, published results from a retrospective study in which they analyzed tumor samples from patients with completely resected lung adenocarcinomas seen at MSKCC from January 2002 to December 2009. ${ }^{15}$ Authors also updated their database, which was entirely made of patients with completely resected lung adenocarcinomas in stage I-III, with identified EGFR mutation. This update had more patients with EGFR mutation in comparison with the prior set. Among 286 patients with resected lung adenocarcinoma and EGFR mutation, 84 patients (29\%) received adjuvant gefitinib or erlotinib. Statistical analysis showed that patients with EGFR-positive lung cancer who received adjuvant TKIs tend to have a longer DFS compared with those who did not receive adjuvant gefitinib or erlotinib $(\mathrm{HR}=0.43, P=0.001$ ). OS rate was numerically superior in the group of patients who received adjuvant TKI, although it was not statistically significant ( $\mathrm{HR}=0.50, P=0.076$ ). The main pitfall of the aforementioned trial is imbalanced. Patients who received adjuvant TKIs had a higher disease stage and were more likely to receive adjuvant chemotherapy before starting adjuvant TKIs. The data from MSKCC are definitely challenging and have great value even if they were collected from a large case series and not from a randomized clinical trial.

\section{Most recent trials evaluating adjuvant TKIs}

At the 2014 ASCO annual meeting, results of two prospective studies designed to evaluate the effect of erlotinib in adjuvant setting in NSCLC were published. The RADIANT trial was a randomized, double-blind, Phase III clinical trial with the aim to investigate efficacy of erlotinib adjuvant treatment in completely resected stage IB to IIIA NSCLC patients who had to have EGFR positivity by immunohistochemistry (IHC) or fluorescence in situ hybridization (FISH). ${ }^{16}$ The flowchart for RADIANT trial is given in Figure 1. Standard adjuvant chemotherapy was optional. Eligible patients were randomized to 2 years of erlotinib or observation. For the primary endpoint of DFS, no significant difference between the two arms was observed. Median DFS was 48.2 months in the placebo arm and 50.5 months in the erlotinib arm. In a subset analysis which included patients with EGFR-mutated disease (161 patients: 102 on erlotinib and 59 on placebo), DFS favored erlotinib in comparison with placebo. Median DFS was 46.4 months for erlotinib and 28.5 months for placebo, but this could not be considered statistically significant because of hierarchical analysis. ${ }^{17}$ This study certainly had limitations and significant bias due to disproportion of stage between two groups, individual usage of adjuvant chemotherapy, and tumor size. While the RADIANT trial was underway, another study had been designed with purpose to give answers about adjuvant treatment with erlotinib, particularly for patients who are positive for EGFR mutation. That was the SELECT trial, which was a single-arm Phase II study of adjuvant erlotinib in resected stage IA to IIIA EGFRpositive NSCLC; ${ }^{18}$ flowchart of SELECT trial is given in Figure 2. In this trial, EGFR positivity had been established by RT-PCR rather than IHC or FISH, which was used in the RADIANT trial. The primary endpoint of the SELECT study was 2 years DFS, which was met with $94 \%$ of patients not having disease recurrence in 2 years' time frame. This study was later expanded to a sample size of 100 patients at seven sites. With median follow-up of 3.5 years, the 2-year DFS was $89 \%$. Median DFS and OS have not been reached. ${ }^{19}$ Two-thirds of all patients completed almost 2-year longterm treatment. It was observed that 24 patients had disease relapse, but only two patients during treatment with erlotinib; the remaining patients had recurrence after stopping the TKI therapy. That observation gave reasons for raising a question about adequate duration of TKI treatment. In addition, 17 patients with disease recurrence were treated with erlotinib in a second round with median treatment time of 10 months. ${ }^{17}$ One potentially pivotal trial was presented on ASCO 2013 by a Chinese group. This trial was different in design than the SELECT study. In that trial, the patients received adjuvant pemetrexed, carboplatin, and gefitinib. ${ }^{20}$ Sixty patients with stage IIIA-N2-positive disease were surgically resected and then randomized in two groups to receive adjuvant pemetrexed and carboplatin with or without gefitinib. This study was terminated early based on a median DFS, which was superior in the group of patients in whom gefitinib had been added to chemotherapy (39.8 months vs 27.0 months, $P=0.014)$. This was a relatively small study in which gefitinib 


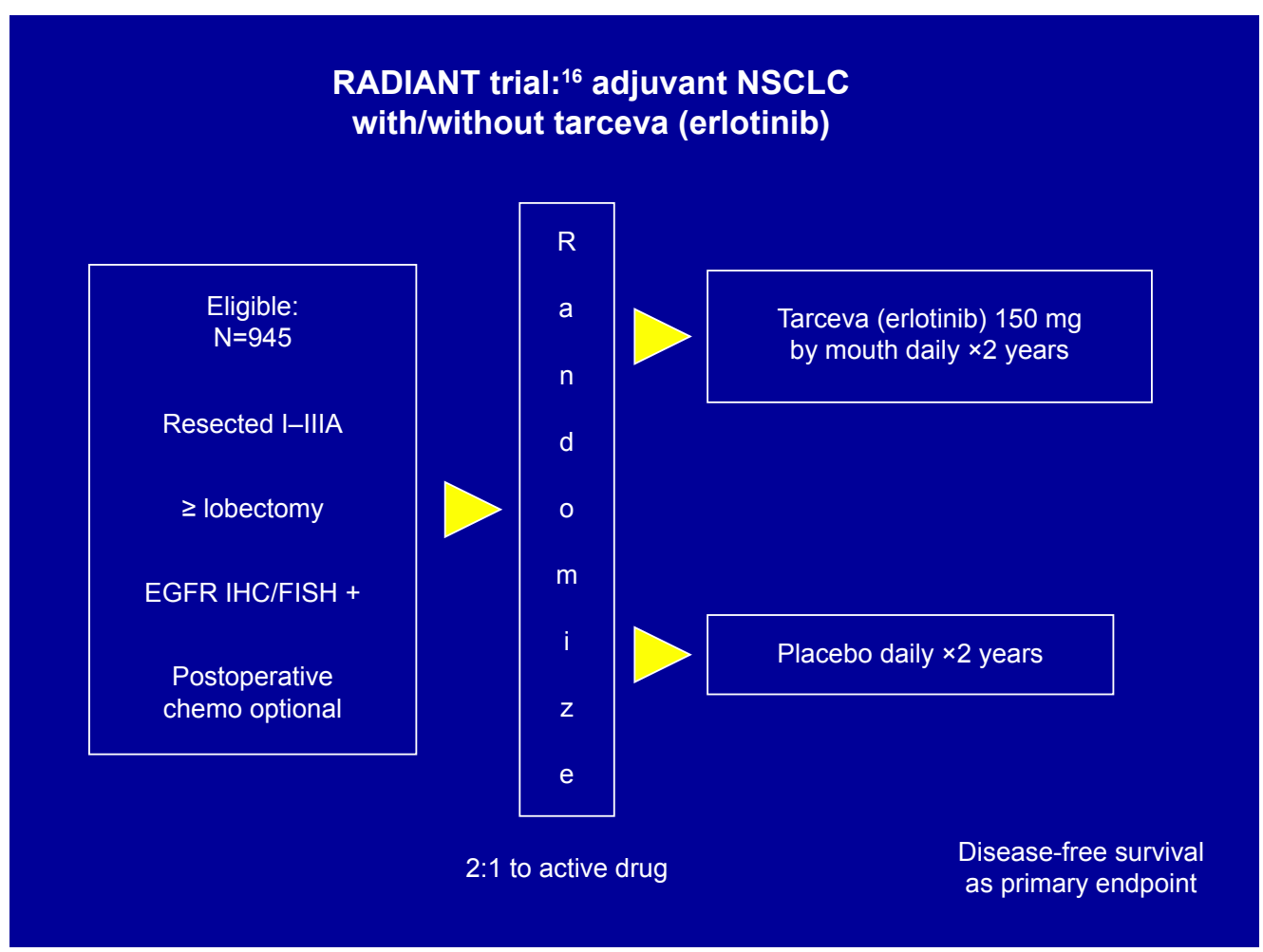

Figure I The flowchart of RADIANT trial.

Abbreviations: NSCLC, non-small-cell lung cancer; EGFR, epidermal growth factor receptor; IHC, immunohistochemistry; FISH, fluorescence in situ hybridization.

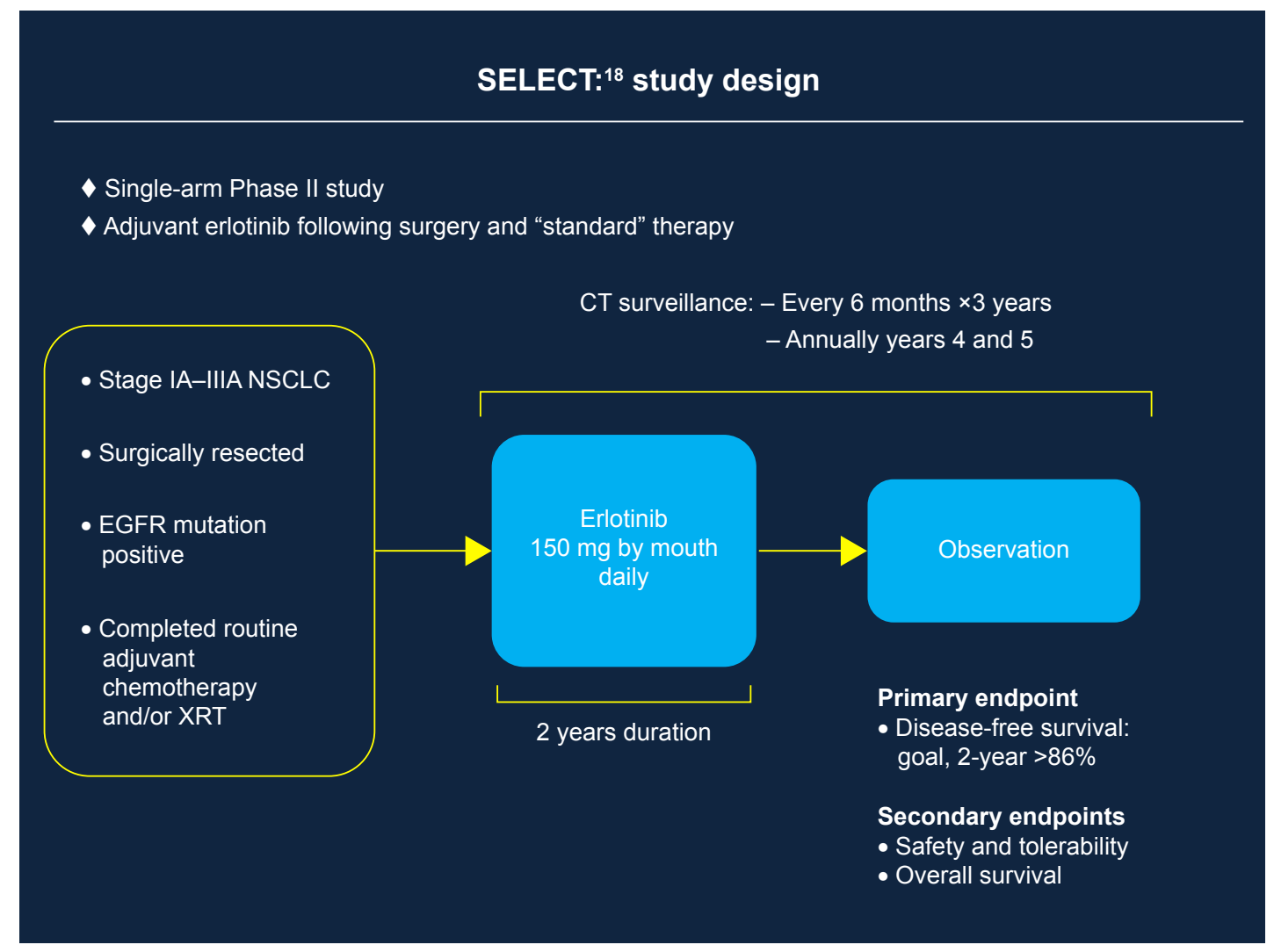

Figure 2 The flowchart of SELECT trial.

Abbreviations: NSCLC, non-small-cell lung cancer; EFGR, epidermal growth factor receptor; CT, computed tomography; XRT, radiation therapy. 
was used in combination with chemotherapy, unlike other studies in which gefitinib was added after finishing standard adjuvant chemotherapy. Nevertheless, these data showed that gefitinib added to standard chemotherapy could delay the development of disease recurrence. Recently, a group of authors from Peking University Cancer Hospital and Institute, Beijing, People's Republic of China, published results from their retrospective study, which included 257 patients with completely resected adenocarcinoma stage I-IIIA. ${ }^{21}$ Among them, 138 patients had EGFR mutation of which 31 patients received adjuvant TKI. Four of them received adjuvant TKI after standard adjuvant chemotherapy, while 27 patients received exclusively TKI therapy with a median treatment duration of 18 months. The EGFR-TKIs included gefitinib, erlotinib, and icotinib. Patients with EGFR-positive mutation who received adjuvant TKIs had longer DFS than those who did not $(P=0.033)$. However, adjuvant TKI therapy did not have an impact on OS between two groups $(P=0.258)$, although patients who received TKIs had better 3-year OS ( $92.5 \%$ vs $81 \%$ ). In this study, 118 patients developed disease recurrence and 64 of them had EGFR-positive disease. Of these 64 patients, eight were on adjuvant TKI treatment and seven of them developed recurrence while they were on treatment with TKIs. Although there was small number of patients who received TKI treatment, this study offered promising results, especially in patients treated with adjuvant EGFRTKI monotherapy. In the same manner, there are currently two studies underway in Asia. In Japan, the WJOG6410L study in patients with stage II and III EGFR-positive NSCLC, patients receive, after surgery, either cisplatin and vinorelbine combination or gefitinib monotherapy. This study has target accrual of 230 patients. ${ }^{21}$ In the People's Republic of China, ADJUVANT trial has a similar design where 220 patients with stage II-IIIA will receive either cisplatin and vinorelbin combination or gefitinib in adjuvant setting. The primary endpoint is DFS and secondary endpoints are OS, 3-year and 5-year DFS rate, 5-year OS rate, adverse events, and quality of life. ${ }^{13}$ Another trial that offers a great opportunity for patients to enter adjuvant trials with new investigational drugs is the ALCHEMIST (A151216) trial. In this trial, patients with stage I-III non-squamous NSCLC are screened for EGFR mutations and ALK rearrangements and then randomized to appropriate targeted therapy (erlotinib or crizotinib) or placebo for 2 years after resection of tumor and standard adjuvant chemotherapy. Patients without EGFR or ALK mutation will be screened for other adjuvant trials investigating new targeted agents. ${ }^{22}$ An overview of discussed trials is provided in Table 1.

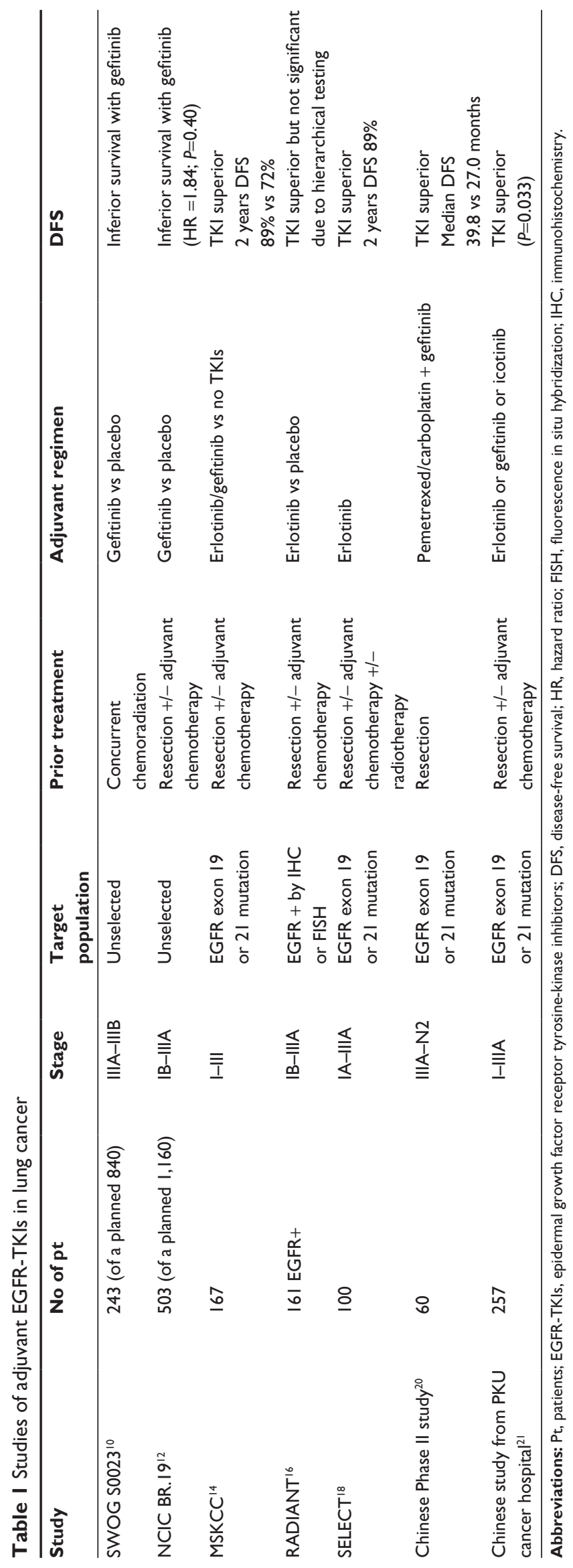




\section{Conclusion}

TKIs in the treatment of advanced non-squamous NSCLC revolutionized therapeutic approach to this disease. There is no doubt about the efficacy of TKI treatment in advanced lung adenocarcinoma. However, the place and role of this treatment in neo-adjuvant or adjuvant setting are still not defined, with many obstacles before introduction in routine treatment. To give or not to give adjuvant EGFR-TKIs still remains questionable. There is a hope that with these latest trials, we will have more definitive answers regarding efficacy of TKIs in adjuvant setting. Right now, we are stumped mostly with small single-arm trials and larger trials; all have limitations such as being terminated early or being conducted in unselected populations without selecting the proper biomarker. There are still a large number of questions that need to be answered. What kind of patients are candidates for adjuvant treatment with EGFR-TKIs? What is the optimal duration of adjuvant TKIs treatment? Is TKI treatment sufficient as a monotherapy or we should use it in combination with standard adjuvant chemotherapy? Do all stages from IA to IIIA have benefit of adjuvant TKI therapy? At the end, there is not enough evidence to give or to avoid adjuvant EGFR-TKIs. One conclusion remains: we should not prescribe these agents in adjuvant setting outside of clinical trials.

\section{Disclosure}

The authors report no conflicts of interest in this work.

\section{References}

1. Jemal A, Bray F, Center MM, Ferlay J, Ward E, Forman D. Global cancer statistics. CA Cancer J Clin. 2011;61(2):69-90.

2. Crino L, Weder W, van Meerbeeck J, Felip E; ESMO Guidelines Working Group. Early stage and locally advanced (non-metastatic) non-small-cell lung cancer: ESMO Clinical Practice Guidelines for diagnosis, treatment and follow-up. Ann Oncol. 2010;21(suppl 5): v103-v115.

3. Pisters KM, Evans WK, Azzoli CG, et al; Cancer Care Ontario; American Society of Clinical Oncology. Cancer Care Ontario and American Society of Clinical Oncology adjuvant chemotherapy and adjuvant radiation therapy for stages I-IIIA resectable non small-cell lung cancer guideline. J Clin Oncol. 2007;25(34):5506-5518.

4. Goldstraw P, Crowley J, Chansky K, et al. The IASLC Lung Cancer Staging Project: proposals for the revision of the TNM stage groupings in the forthcoming (seventh) edition of the TNM classification of malignant tumours. J Thorac Oncol. 2007;2(8):706-714.

5. Zaric B, Stojsic V, Tepavac A, et al. Adjuvant chemotherapy and radiotherapy in the treatment of non-small cell lung cancer (NSCLC). J Thorac Oncol. 2013;5(suppl 4):S371-S377.

6. Zhou C, Wu YL, Chen G, et al. Erlotinib versus chemotherapy as first-line treatment for patients with advanced EGFR mutation-positive non-smallcell lung cancer (OPTIMAL, CTONG-0802): a multicentre, open-label, randomised, phase 3 study. Lancet Oncol. 2011;12(8):735-742.
7. Rosell R, Carcereny E, Gervais R, et al; Spanish Lung Cancer Group in collaboration with Groupe Français de Pneumo-Cancérologie and Associazione Italiana Oncologia Toracica. Erlotinib versus standard chemotherapy as first-line treatment for European patients with advanced EGFR mutation-positive non-small-cell lung cancer (EURTAC): a multicentre, open-label, randomised phase 3 trial. Lancet Oncol. 2012;13(3):239-246.

8. Slamon D, Eiermann W, Robert N, et al; Breast Cancer International Research Group. Adjuvant trastuzumab in HER2-positive breast cancer. N Engl J Med. 2011;365(14):1273-1283.

9. Dematteo RP, Ballman KV, Antonescu CR, et al; American College of Surgeons Oncology Group (ACOSOG) Intergroup Adjuvant GIST Study Team. Adjuvant imatinib mesylate after resection of localised, primary gastrointestinal stromal tumour: a randomised, double-blind, placebo-controlled trial. Lancet. 2009;373(9669):1097-1104.

10. Kelly K, Chansky K, Gaspar LE, et al. Phase III trial of maintenance gefitinib or placebo after concurrent chemoradiotherapy and docetaxel consolidation in inoperable stage III non-small-cell lung cancer: SWOG S0023. J Clin Oncol. 2008;26(15):2450-2456.

11. Thatcher N, Chang A, Parikh P, et al. Gefitinib plus best supportive care in previously treated patients with refractory advanced nonsmall-cell lung cancer: results from a randomised, placebo-controlled, multicentre study (Iressa Survival Evaluation in Lung Cancer). Lancet. 2005;366(9496):1527-1537.

12. Goss GD, O'Callaghan C, Lorimer I, et al. Gefitinib versus placebo in completely resected non-small-cell lung cancer: results of the NCIC CTG BR19 study. J Clin Oncol. 2013;31(27):3320-3326.

13. Zhai H, Zhong W, Yang X, Wu YL. Neoadjuvant and adjuvant epidermal growth factor receptor tyrosine kinase inhibitor (EGFR-TKI) therapy for lung cancer. Transl Lung Cancer Res. 2015;4(1):82-93.

14. Janjigian YY, Park BJ, Zakowski MF, et al. Impact on disease-free survival of adjuvant erlotinib or gefitinib in patients with resected lung adenocarcinomas that harbor EGFR mutations. J Thorac Oncol. 2011;6(3):569-575.

15. D'Angelo SP, Janjigian YY, Ahye N, et al. Distinct clinical course of EGFR-mutant resected lung cancers: results of testing of 1118 surgical specimens and effects of adjuvant gefitinib and erlotinib. J Thorac Oncol. 2012;7(12):1815-1822.

16. Kelly K, Altorki NK, Eberhardt WEE. A randomized, double-blind phase 3 trial of adjuvant erlotinib (E) versus placebo (P) following complete tumor resection with or without adjuvant chemotherapy in patients (pts) with stage IB-IIIA EGFR positive (IHC/FISH) non-small cell lung cancer (NSCLC): RADIANT results. J Clin Oncol; 2014; 32(5): Abstract 7501.

17. Shepherd FA, Altorki NK, Eberhardt WEE. Adjuvant erlotinib (E) versus placebo (P) in non-small cell lung cancer (NSCLC) patients (pts) with tumors carrying EGFR-sensitizing mutations from the RADIANT trial. J Clin Oncol. 2014;32(15):Abstr7513.

18. Pennell NA, Neal JW, Chaft JE. SELECT: a multicenter phase II trial of adjuvant erlotinib in resected early-stage EGFR mutation-positive NSCLC. J Clin Oncol. 2014;32(5s):Abstr7514.

19. Lampaki S, Lazaridis G, Zarogoulidis K, et al. Defining the role of tyrosine kinase inhibitors in early stage non-small cell lung cancer. $J$ Cancer. 2015;6(6):568-574.

20. Li N, Ou W, Ye X, et al. Pemetrexed-carboplatin adjuvant chemotherapy with or without gefitinib in resected stage IIIA-N2 non-small cell lung cancer harbouring EGFR mutations: a randomized, phase II study. Ann Surg Oncol. 2014;21(6):2091-2096.

21. Lv C, An C, Feng Q, et al. A retrospective study of stage I to IIIa lung adenocarcinoma after resection: what is the optimal adjuvant modality for patients with an EGFR mutation? Clin Lung Cancer. Epub 2015 April 20.

22. Govindan R. Lung cancer: molecular profiling and genomics. Extended education session. Presented at: ASCO Annual Meeting; 31 May-3 June 2013; Chicago, IL, USA. 
OncoTargets and Therapy

\section{Publish your work in this journal}

OncoTargets and Therapy is an international, peer-reviewed, open access journal focusing on the pathological basis of all cancers, potential targets for therapy and treatment protocols employed to improve the management of cancer patients. The journal also focuses on the impact of management programs and new therapeutic agents and protocols on

patient perspectives such as quality of life, adherence and satisfaction The manuscript management system is completely online and includes a very quick and fair peer-review system, which is all easy to use. Visit http://www.dovepress.com/testimonials.php to read real quotes from published authors.

Submit your manuscript here: http://www.dovepress.com/oncotargets-and-therapy-journal 